\title{
MAQUINARIA PARA LA PRODUCCION DE CONSTRUCCIONES EN TIERRA
}

(MACHINERY FOR THE PRODUCTION OF EARTH CONSTRUCTIONS)

Juan Diaz Romeral (V.M.B.C.)

869-15

\section{PRENSAS MANUALES}

\section{UNATA (Bélgica)}

Es una máquina sencilla con la que se trabaja de modo totalmente manuales, debe ser fijada sobre una superficie de madera y trabajar sobre un suelo previamente compactado. La presión sobre el molde se efectúa mediante un pistón que es comprimido por la fuerza física de una persona.

Dimensiones del bloque: $29 \times 14 \times 9 \mathrm{~cm}$. Rendimiento: $20-60$ bloques/hora con un equipo de 2-6 personas.

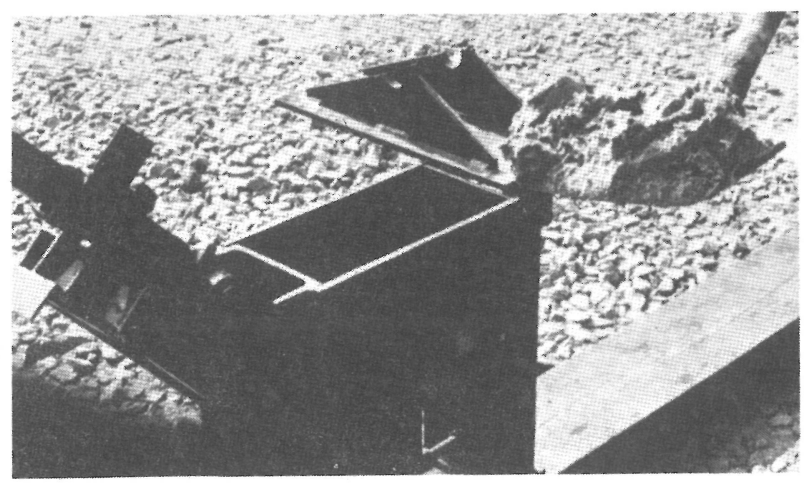

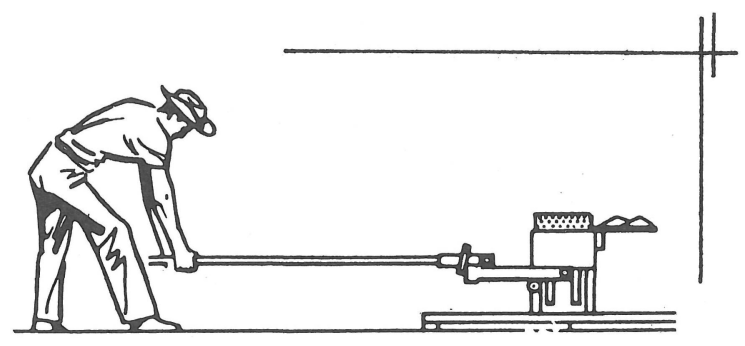

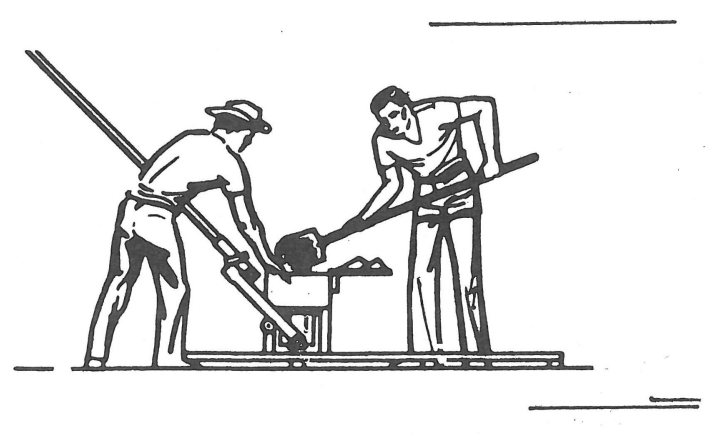




\section{TERSTARAM (PLATBROOD. Bélgica)}

Prensa manual para moldeo de tierra por compresión. Idónea para fabricación de ladrillos de tierra estabilizada o ladrillos de tierra cruda. Admite el diseño de moldes de hasta $29,5 \times 29,5$ $\mathrm{cm}$. Diseñada por su robustez especialmente para trabajos en paises en vías de desarrollo.

Rendimiento de 800 bloques diarios de $29,5 \times 14$ $\times 9 \mathrm{~cm}$. o bien de $22 \times 11 \times 6 \mathrm{~cm}$.

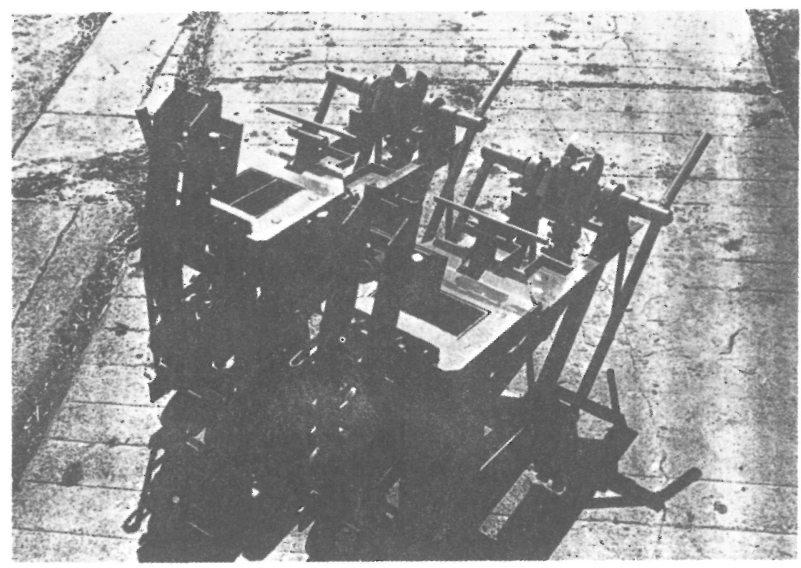

\section{CERAMAN (CERATEC. Bélgica)}

Esta prensa permite un completo funcionamiento mecánico. La mesa rotatoria consta de 3 moldes respectivamente rellenados, compactados y desalojados al mismo tiempo en las diferentes posiciones de la máquina. La fuerza motriz asegura la presión y desmoldeado continuo de los bloques. La mano de obra se utiliza mecánicamente para la carga de la materia prima y transporte del bloque acabado. La tierra utilizada no necesita preparación anticipada, la maquinaria asociada a un triturador que permite una producción elevada con medios reducidos.

Dimensiones del bloque: $22 \times 10,7 \times 7 \mathrm{~cm}$. Producción horaria: 600-800 ladrillos.

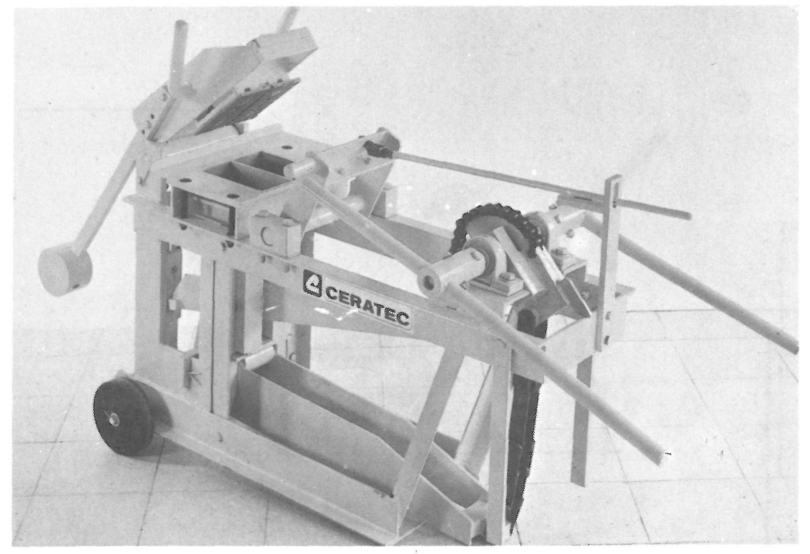

\section{BREPAK (Welding Industries. Inglaterra)}

La prensa manual multibloc BREPAK comprime una cavidad de dimensiones fijas, que junto con toda la estructura del soporte forma una unidad completa metálica. Toda la unidad puede montarse sobre una cimentación permanente o bien sobre una base de madera que permita su transporte. La presión sobre el bloque de tierra se aplica mediante un gato hidráulico que proporciona una presión de 8000 p.s.i. (libras pulgada cuadrada $10 \mathrm{MN} / \mathrm{m}^{2}$ ) y que asegura una compactación completa del bloque.

Dimensiones del bloque: $29 \times 14 \times 10 \mathrm{~cm}$. Rendimiento $35 / 40$ bloques/hora con un equipo de 5-6 operarios.

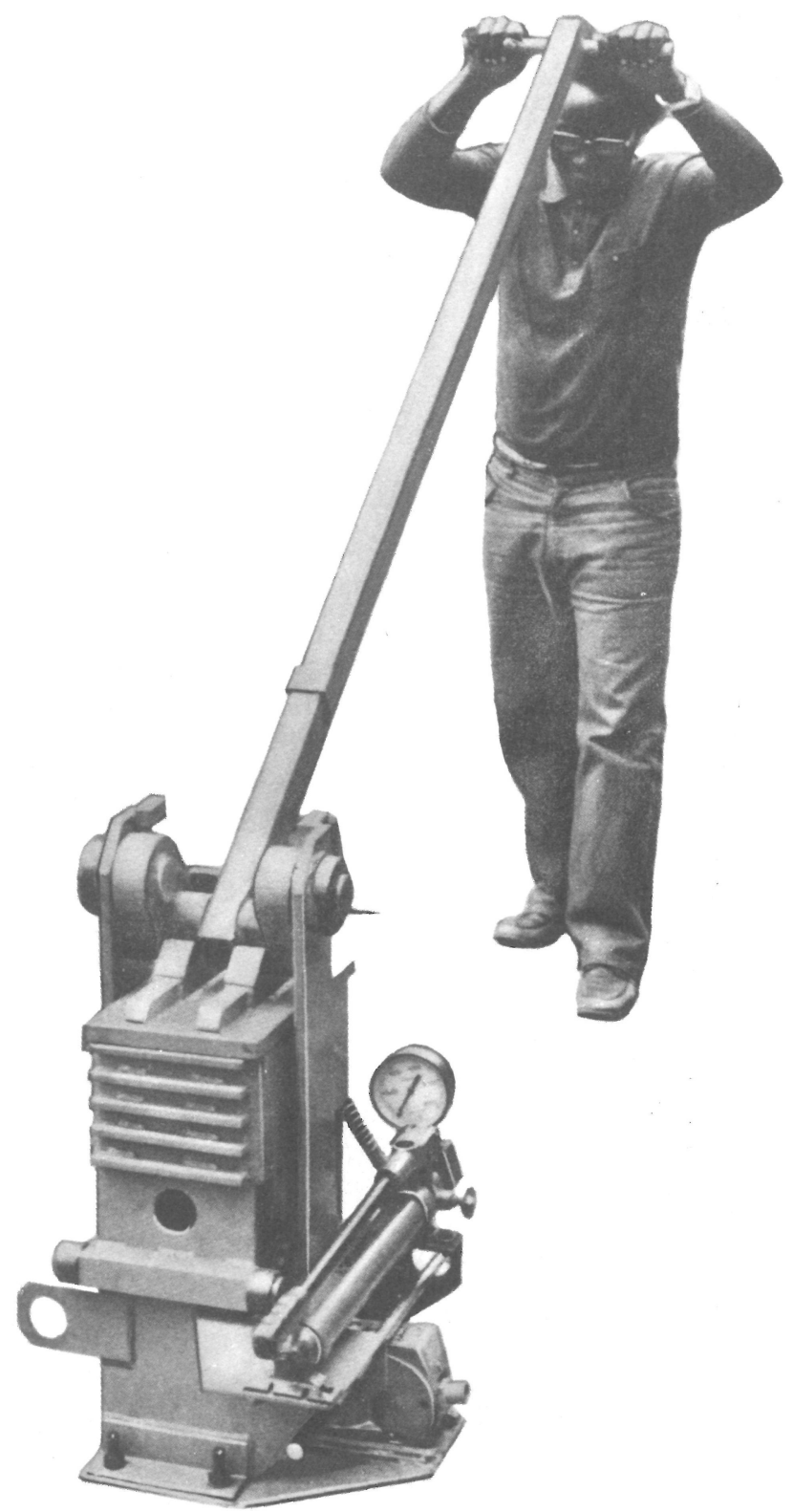




\section{TRITURADORAS}

\section{CERADES HZ (CERATEC. Bélgica)}

Especialmente diseñada para ser usada junto con la prensa manual CERAMAN o con la prensa automática CERAMATIC. Se utiliza principalmente para la rotura, mezcla y homogeneización del material cerámico crudo de dureza media, con una humedad natural del 0-25\% y una dureza de $40 \mathrm{~Hz}$ de 1 a 3. La fina granulometria de la arena preparada es idónea para el completo llenado de los moldes y asegura una producción de bloques de alta calidad.

\section{Tamizadora CATAPULTER (CONSOLID A.G. Suiza)}

Mediante un motor de gasolina, esta máquina trabaja no sólo con suelo seco, especialmente cuando se encuentra húmedo, esta tamizadora trabaja muy adecuadamente. Mediante cuatro pasos combinados de desmenuzado, mezclado, oreado y limpieza, representa una importante ventaja sobre otros métodos de preparación de suelos.

\section{Pulverizadora \\ (Sistema CARR. PLATBOROOD. Bélgica)}

Esta pulverizadora manual se acciona mediante dos maniveles, dos discos giran en sentido inverso mediante una transmisión por correas, la tierra que accede en medio de los dos discos debe atravesar dos hileras de barrotes que la pulverizan. Este sistema tiene la ventaja de pulverizar cualquier tipo de tierra, incluso laterita, su inercia permite un trabajo continuo sin esfuerzo una vez la máquina entra en funcionamiento.
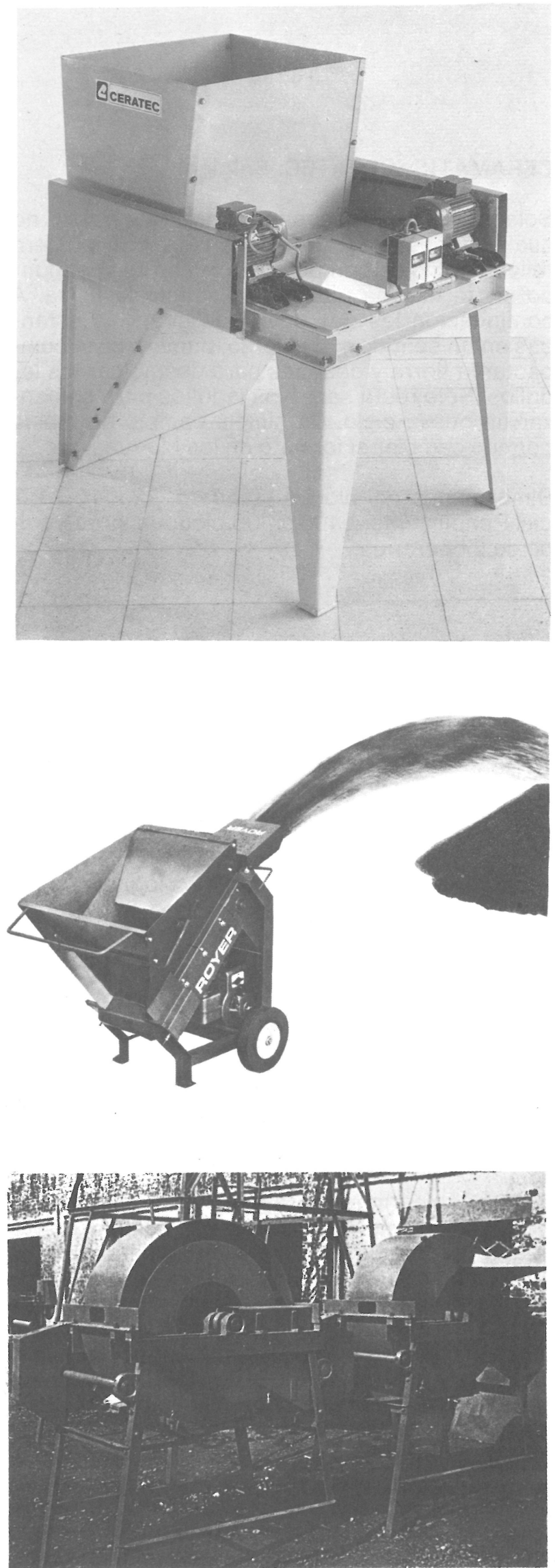


\section{PRENSAS AUTOMATICAS}

\section{CERAMATIC (CERATEC. Bélgica)}

Solamente son necesarios dos operarios no cualificados para su funcionamiento. El primero rellena el molde con una pala mientras el segundo cierra la tapa que pre-compacta la tierra. A continuación los dos operarios giran las palancas en un sentido y al inverso, primero para compactar la tierra y después para desmoldar los ladrillos. Sólo resta sacar los ladrillos para comenzar un nuevo ciclo. Un simple cambio de molde permite cambiar el formato de los bloques.

Dimensiones del bloque standard: $22 \times 10,7 \times 7$ $\mathrm{cm}$. Rendimiento: $1400-2000$ bloques: hora equipo de 2 operarios.

\section{SEMI-TERSTAMATIQUE (PLATBROOD. Bélgica)}

Su concepción permite la utilización por operarios pero cualificados y su entretenimiento realizado por artesanos locales sin formación especializada. Los materiales de fabricación permiten un uso intensivo y su robusta fabricación la hacen idónea en condiciones climatológicas extremas (calor, humedad, fuerte viento con arena). Permite un rendimiento diario de 500 ladrillos de $22 \times 10,5 \times 6 \mathrm{~cm}$. o bien 2.500 de $29,5 \times 14 \times$ $9 \mathrm{~cm}$.
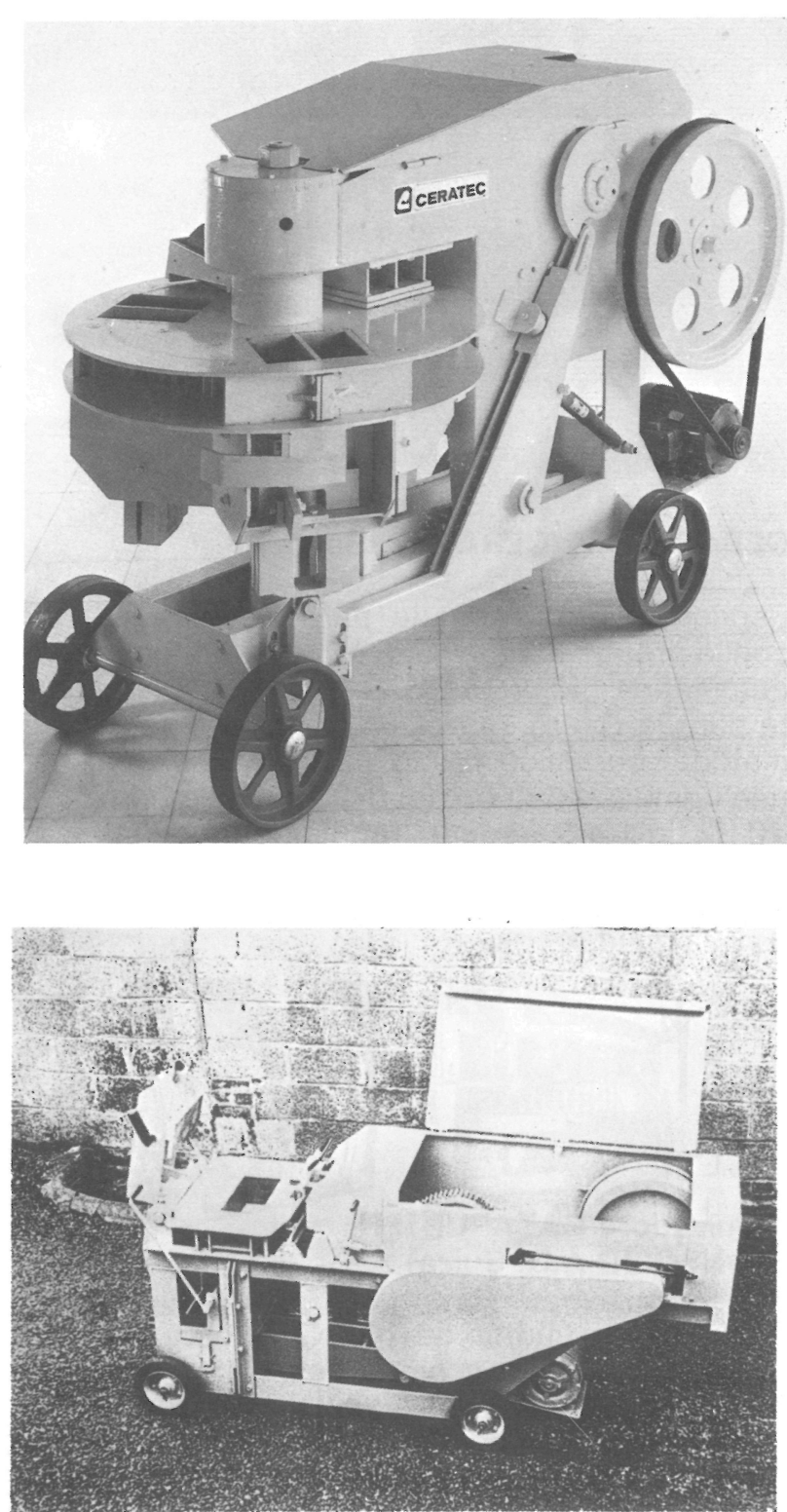

\section{UNIDADES COMPLETAS DE FABRICACION}

\section{CLU. 3000 (CONSOLID A.G. Suiza)}

Esta máquina móvil para ladrillos de adobe permite, una vez suministrado en ella el suelo pulverizado, y después de un tiempo de mezclado aproximado de $1 \mathrm{~min}$. una producción por carga de 20-25 ladrillos según el tipo de suelo. Los moldes se llenan automáticamente con la tierra proporcionada. El giro de la mesa por un cuarto cada vez es accionado por un operario para llevar el molde lleno debajo del cabezal de la prensa. Utilizando la palanca el operario prensa el molde lleno formando un ladrillo bien compactado. Simultáneamente el ladrillo anterior prensado es expulsado para ser luego apilado. Un equipo de 4-5 operarios puede producir 3.000-4.000 ladrillos diarios de dimensiones $25 \times 12 \times 7,5 \mathrm{~cm}$.

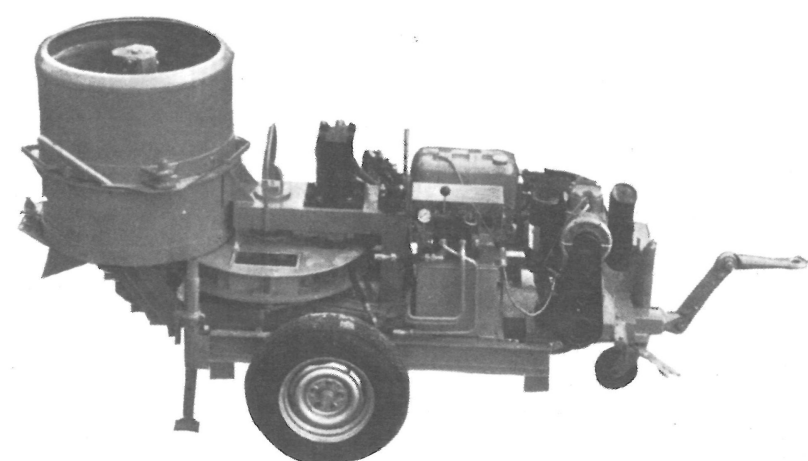

